\title{
Criminologie
}

\section{Comment évaluer l'impact du travail des policiers de proximité ?}

\section{Frédéric Ocqueteau}

Volume 36, numéro 1, printemps 2003

Police et prévention : évaluation et analyse d'impact

URI : https://id.erudit.org/iderudit/006556ar

DOI : https://doi.org/10.7202/006556ar

Aller au sommaire du numéro

\section{Éditeur(s)}

Les Presses de l'Université de Montréal

ISSN

0316-0041 (imprimé)

1492-1367 (numérique)

Découvrir la revue

Citer cet article

Ocqueteau, F. (2003). Comment évaluer l'impact du travail des policiers de proximité ? Criminologie, 36(1), 121-141. https://doi.org/10.7202/006556ar
Résumé de l'article

Le présent article rend compte des réflexions théoriques et empiriques menées au sujet des préalables indispensables pour évaluer l'impact de la réforme de la «police de proximité» instaurée en France en 1998. Il montre l'importance, pour juger de l'efficience de la réforme et de son éventuelle efficacité, d'avoir une bonne connaissance des systèmes d'action concrets locaux et des divers intérêts qui y sont défendus par ceux qui les mettent en oeuvre.

Accessoirement, il montre comment l'exercice de dévoilement peut constituer un danger politique dans la mesure où il peut s'avérer déstabilisant pour ceux-là mêmes qui ont intérêt à revendiquer le succès national de la réforme avant même qu'elle soit achevée et que l'impact à long terme puisse être même diagnostiqué. Il montre pourquoi l'évaluation de nouvelles orientations policières n'est pas qu'une simple affaire de technique managériale et d'indicateurs chiffrés. 


\title{
Comment évaluer l'impact du travail des policiers de proximité?
}

\author{
Frédéric 0 cqueteau \\ CNRS - CERSA \\ France \\ focqueteau@u-paris2.fr
}

RÉSUMÉ - Le présent article rend compte des réflexions théoriques et empiriques menées au sujet des préalables indispensables pour évaluer l'impact de la réforme de la "police de proximité» instaurée en France en 1998. II montre l'importance, pour juger de l'efficience de la réforme et de son éventuelle efficacité, d'avoir une bonne connaissance des systèmes d'action concrets locaux et des divers intérêts qui y sont défendus par ceux qui les mettent en œuvre. Accessoirement, il montre comment l'exercice de dévoilement peut constituer un danger politique dans la mesure où il peut s'avérer déstabilisant pour ceux-là mêmes qui ont intérêt à revendiquer le succès national de la réforme avant même qu'elle soit achevée et que l'impact à long terme puisse être même diagnostiqué. II montre pourquoi l'évaluation de nouvelles orientations policières n'est pas qu'une simple affaire de technique managériale et d'indicateurs chiffrés.

ABSTRACT - The article delineates the theoretical and empirical requirements for assessing the overall impact of a community policing reform initiated in 1998. To decide about its effectiveness and its efficiency, I argue that it is necessary to understand thoroughly the dynamics of local «systems of action» and to provide a grounded and contextual analysis of the variety of self-interests pursued by those who have been ordered to implement the reform. I also show that the empirical task of uncovering the dynamics of self-interests and their impact on evaluation outcomes may be viewed as a threat for «political reformers» who have publicly gambled on the national success of the reform even before a formal assessment of its impact has been conducted and before a plausible evaluation of the generalizability of experimental sites findings has been undertaken. The paper argues why the stakes of evaluation strategies of this new form of policing cannot be addressed only as a technical managerial or methodological issue. 
M ême si pour les sociologues (Becker, 2002) les ficelles du «comment» sont souvent plus prometteuses, en termes d'approfondissement de la connaissance scientifique ou de dépassement de la pensée conventionnelle, que celles du «pourquoi », on n'échappe pas facilement à un certain nombre de prérequis liminaires pour répondre à la question apparemment simple du titre. Ils exigent que soient levées au moins plusieurs hypothèques: qui (se) pose cette question? de quelle sorte de missions policières à évaluer parle-t-on exactement? à quel niveau (ou sur qui) situe-t-on exactement l'impact des réformes mises en œuvre? de quels outils se dote-t-on pour les mesurer? J'essaierai de les lever une à une en me situant dans le cadre français de la réforme dite de «police de proximité» et de montrer dans quel contexte des évaluations éparses ont pu voir le jour. D'une part, parce que les questions d'évaluation ont commencé à pénétrer la conscience des réformateurs institutionnels et celle de la Cour des comptes comme un horizon de pratiques légitimes en vue d'améliorer la "qualité du service public»; d'autre part, parce qu'en tant que sociologue ayant piloté des recherches durant quatre ans au ministère de l'Intérieur à ce sujet j'ai été amené, ou bien à commanditer et engranger des études finalisées, ou bien à enquêter personnellement sur ces questions. Cette position de transfuge (0 cqueteau, 2002 : 2-3) expliquera en grande partie pourquoi des interrogations internes et extérieures à l'institution, et apparemment communes, au sujet de la notion de «performance» policière m'ont paru pouvoir se recouper sans véritablement s'entendre, ni hélas toujours bien se comprendre.

\section{Pressions aux calculs de "performance » des institutions de sécurité réformées}

II convient d'abord de garder présent à l'esprit l'idée que les services de la sécurité intérieure ont sans doute été les derniers services publics en France à faire l'objet d'une réflexion sur le suivi du «coût global » de leur fonctionnement, et ce n'est que très récemment que l'on en a pris conscience afin de mieux piloter les administrations centrales (police, justice, administration pénitentiaire). Cela signifiait à toutes fins pratiques de vouloir contribuer à une meilleure allocation de leurs moyens humains et budgétaires sur le territoire national en rapport aux performances attendues des agences contributrices de sécurité. II n'existait au sein du 
ministère de l'Intérieur aucune culture spécifique du management public, et ce bien qu'aux débuts de la $V^{\text {e }}$ R épublique une ordonnance du 2 janvier 1959, restée lettre morte, ait suggéré qu'avant le vote de tout nouveau crédit chaque ministère dut faire l'effort de présenter un rapport annuel d'efficacité avec la loi de règlement précisant «objectifs, résultats attendus et obtenus, indicateurs et coûts associés» (Pierre, 2001). Si les approches budgétaires restent dans notre pays en grande partie organiques, la méthode analytique du calcul des coûts commence à avoir ses adeptes dans la mesure où ils pensent qu'elle permettrait un meilleur raisonnement sur l'efficacité, entendue de deux manières: au sens large, comme susceptible de dégager des gains de productivité au sein des services publics de l'État dans leur ensemble. Au sens plus restreint de la "sécurité intérieure», comme permettant un inventaire annuel des moyens humains, matériels, immobiliers de chaque sous-administration productrice de sécurité dans le total des dépenses, mis en perspective avec les financements de la sécurité civile par les collectivités locales, ceux des services de sécurité des grandes entreprises publiques (les transports collectifs notamment) et ceux des coûts liés aux dépenses de sécurité privée. $M$ ais cette logique de comptabilité managériale liée à la productivité des services de sécurité (C rawford, 1997) ne saurait évidemment suffire. Car si la sécurité a un coût, l'« insécurité», ou plutôt l'absence ou la diminution de la sécurité, en a un autre qui demande à être mis en balance dans les grands équilibres macro-économiques. À la suite de certains économistes des coûts du crime, C. Pierre (2001: 59 et suivantes) suggère à ce sujet de tenir compte des coûts privés directs (sociaux, psychologiques et économiques, dont une partie est prise en charge par les compagnies d'assurance), des coûts publics directs (dégâts causés par des manifestations protestataires mal encadrées se traduisant par des frais de contentieux et de réparation civile pris en charge par le contribuable, par exemple), des coûts privés indirects (dégradation de l'environnement nuisant aux activités économiques et à la croissance...) et des coûts publics indirects (expansion de l' «économie illégale» ou ralentissements de l'activité économique liés au contexte d'insécurité affectant certaines zones du territoire). II aurait pu y ajouter de façon moins restrictive le gigantesque océan des dégâts de la criminalité en col blanc et de la fraude fiscale, dont il estime peut-être qu'elle ne fait pas partie d'une insécurité sur laquelle police et justice auraient quelque prise. 
La réforme dite de la «police de proximité», annoncée lors du colloque de Villepinte d'octobre 1997, est venue conforter les procédures d'un autre dispositif partenarial entre maires, préfets ${ }^{1}$ et procureurs et divers acteurs concernés, au sein des villes affectées par l'insécurité, celui des «contrats locaux de sécurité» (ministère de l'Emploi et de la Solidarité et al., 1998). En cette occasion, il fut décidé qu'une cellule de suivi accompagnerait les étapes de la réforme et que des évaluations libres de chercheurs de l'Institut des hautes études de la sécurité intérieure (IHESI) pourraient s'y adjoindre pour aider à généraliser le dispositif à partir des premières expérimentations locales. Rappelons ici quelques éléments de contexte indispensables pour montrer qu'il s'est bien agi, au moins du point de vue de la doctrine, de l'une des plus importantes réformes de la police de l'après-guerre.

Trois grands objectifs clairement affichés et cinq modalités d'action principales ont constitué l'essentiel de l'argument (ministère de l'Intérieur, 2000): tendre vers une police qui sache anticiper et prévenir les difficultés liées à la délinquance urbaine; construire une police qui connaisse son territoire et la population qui y vit, population qui sache elle-même connaître et reconnaître sa police; construire une police qui réponde mieux aux attentes des citoyens. Q uant aux modalités d'action opérationnelles, elles sont basées sur le diagnostic de la crise de l'îlotage traditionnel auquel, pour porter remède, il convenait de mieux implanter la police dans des «secteurs» préoccupants au sein de chaque circonscription; de lui faire rechercher un contact permanent avec la population en facilitant la réception et la prise en charge effective de ses plaintes et demandes; de former des policiers polyvalents à tous les niveaux de la hiérarchie; de les responsabiliser et les professionnaliser dans le sens de la citoyenneté et de les mettre en état de rendre un service de qualité.

Concrètement, les premières expérimentations pilotes de cette réforme ont démarré dans cinq circonscriptions françaises en 1999 (Beauvais, Châteauroux, les Ulis, N îmes et Garge-lès-Gonesse), puis furent étendues six mois plus tard (1999-2000) à 67 circonscriptions (couvrant deux millions de citoyens), la phase de généralisation à l'ensemble du territoire devant concerner à terme les deux tiers des Français (20012003). N otons que cette réforme affecte également les brigades de

1. En France, c'est le représentant officiel de l'État central à l'échelon départemental (au nombre de 95). 
gendarmerie en charge des «zones rurbaines» (rurales-urbaines, situées à la périphérie des villes) à l'égard de qui l'on procéda également à des tests sur des villes où il n'existait pas de commissariats de police (en 2003, son champ d'action couvrira le tiers restant des habitants du territoire). Elle concerne donc pour l'essentiel les effectifs de la Direction générale de la police nationale et notamment ceux affectés à la sécurité publique, y compris les effectifs de la Préfecture de police de Paris (donc 60000 policiers environ au total). D 'autres directions et services centraux sont concernés, tels les RG (renseignements généraux) et les CRS (compagnies républicaines de sécurité), mais dans une moindre mesure. Les CRS ont par exemple été pensées comme venant en renfort des agents de police de proximité (ils sont censés se concentrer au minimum quatre mois sur certains centres urbains très problématiques et y être déployés en petites unités).

La conceptualisation et la fabrication, par la cellule «Ville et banlieues» de la Direction centrale des renseignements généraux, d'une échelle statistique dite des "violences urbaines» dans les "quartiers sensibles» de certaines villes problématiques (1 200 répertoriés en France) a facilité l'élaboration du référentiel commun à l'ensemble de ces polices ( 0 cqueteau et al., $2002: 26$ et suivantes).

Dans le domaine de la logistique, la mise en place de la réforme a nécessité d'importants moyens financiers pour ouvrir et équiper des centaines de nouveaux postes, bureaux et points de contacts de police. Enfin, un important travail de formation initiale et continue dans les seize écoles de police françaises est en cours et doit se poursuivre dans les trois ans.

II apparut assez vite, sous couvert d'évaluer l'ensemble de cette réforme (Ferret et 0 cqueteau, 1998), que nos conseils furent correctement intégrés dans les travaux interministériels, puisque ceux-ci cherchèrent bien à mobiliser trois concepts disponibles avec les indicateurs censés en mesurer les différentes dimensions pour écl airer le Parlement. IIs durent toutefois conclure très vite à la difficulté de l'entreprise, puisque la plupart des indicateurs n'étaient pas disponibles sur le marché. En reprenant en effet la notion d'efficience (comme le rapport entre moyens engagés et résultats obtenus), celle d'impact ou d'efficacité socio-économique (comme la capacité à modifier l'environnement social, ou résultats obtenus in fine) et de qualité des services (comme la prise en compte de la demande des usagers), notions dont l'interaction était censée produire une juste mesure de performance, il fallut en effet rapidement déchanter. Tout simplement parce 
que la pression à réformer l'institution policière le temps d'une législature, pour satisfaire les exigences du ministre promoteur de la réforme, fit qu'on oublia en cours de route un certain nombre de l'ensemble des prérequis bien perçus initialement. Et ce en dépit du fait, par exemple, que s'agissant de l'autre réforme interministérielle des contrats locaux de sécurité (CLS), I'IHESI avait lui-même joué, par le biais de son département d'ingénierie, un rôle moteur d'analyste et de conseiller en élaborant une méthodologie de base pour les diagnostics et en en prenant en charge une vingtaine, préalablement à la signature des contrats (ministère de l'Emploi et de la Solidarité et al., 1998; Chalumeau et Glorieux, 1999). II s'avéra impossible d'évaluer correctement l'impact de la réforme de la nouvelle police, à part quelques exceptions sur lesquelles je reviendrai, puisqu'on n'avait à l'évidence pas le temps d'y sacrifier ni de tirer d'enseignements significatifs sur les sites pilotes vu la pression politique à la généraliser, et c'est donc à marche forcée que l'on s'ingénia à le faire à moyens quasi constants. Les corps d'inspection se contentèrent simplement d'observer que, dans chacune des circonscriptions, l'égalité de traitement en allocations de moyens supplémentaires était de mise: ils ne pratiquèrent tout au plus qu'une analyse de l'efficience dans toutes les circonscriptions de sécurité publique de l'H exagone.

\section{Pressions au rapprochement des indicateurs de connaissance de l'insécurité}

Un travail de réflexion avait pourtant été intensivement consenti depuis plusieurs années dans les bureaux d'étude sur l'harmonisation des indicateurs de connaissance de l'insécurité et de la qualité de la vie urbaine pour tenter de sortir l'appareil policier de la situation sans issue dans laquelle il est enfermé depuis des lustres et que connaît tout étudiant en criminologie. Je veux parler du piège de l'«index 4001 », nom de l'outil statistique fabriqué par la police et la gendarmerie depuis 1972, qui donne à la fois une mesure locale et nationale de l'évolution annuelle des crimes et des délits, et une mesure du taux de leur élucidation par rapport aux plaintes enregistrées (conjointement avec la Gendarmerie depuis 1985). Q uelles que soient les critiques classiques portées à cet instrument de mesure statistique des délinquances qui ne révèlerait en définitive qu'une mesure de l'activité proactive et réactive du travail policier et non une mesure de la criminalité réelle voire apparente (Robert, 1977), I'administration et des chercheurs continuent à s'en servir, y 
compris pour la première pour réfléchir aussi à un meilleur redéploiement des effectifs policiers et gendarmiques en vue de leur plus grande efficacité opérationnelle sur le terrain (voir, par exemple, la réforme avortée de 1999 faisant suite au rapport Carraz-H yest, 1998).

II n'en reste pas moins que l'administration française a fini par se convaincre que cette source n'était pas entièrement satisfaisante pour comprendre les liens existants entre la victimation réelle des citoyens et leur besoin d'être rassurés (autre formulation grossière de la notion de "demande de protection des personnes et de leurs biens»). De toute évidence, les enquêtes national es de victimation ne sont pas encore devenues des outils opérationnels pour la police, comme elles le sont par exemple en Catalogne (Ferret et $M$ affre, 2000) ou en Belgique pour les villes qui ont signé un contrat de sécurité, tandis que les enquêtes de délinquance auto-confessée (B ègue, 2000 ; R oché, 2001) semblent être mieux entendues, même si elles sont encore loin d'être routinisées en raison de leurs coûts prohibitifs. II se trouve que l'enquête locale de Roché, ayant fait ressortir chez les jeunes lycéens de 13 à 19 ans interrogés un noyau dur de $5 \%$ d'entre eux révélant $65 \%$ des faits de délinquance, est venue conforter le savoir profane des policiers. Cet analyste ayant assuré par ailleurs que des coefficients de corrélation majeurs entre trois variables («qualité de l'exercice du contrôle parental», "absentéisme scolaire» et «fraudes dans les transports») annonçaient une forte propension à l'entrée dans un cycle de délinquance caractérisé, I'occasion était politiquement inespérée pour ancrer encore un peu plus dans leur bien-fondé des projets récurrents de réouverture de maisons de correction pour mineurs, à visée neutralisatrice.

La suspicion en instrumentalisations réciproques entre chercheurs et politiques est en général d'autant plus de mise que les sociologues mobilisés n'entendent pas eux-mêmes aller jusqu'au bout de leur démarche quand ils se refusent par exemple, ce qui est leur droit, à donner à la police des «ficelles» pour améliorer un service public paraissant toujours prioritairement orienté vers la répression de certaines populations (Jobard, 2002). Q uand ils s'essaient explicitement de construire des outils compatibles avec les nomenclatures policières, les résultats paraissent bien décevants. Une étude (G rémy, 2000) a montré, par exemple, que le volume de la délinquance révélée par une enquête nationale de victimation financée par I'IHESI et administrée par l'IN SEE en janvier 1999 était compatible avec les index de la police uniquement sur les infractions de cambriolages, de vols de véhicules et de vols sans violence. Elle ne parve- 
nait pas en revanche à établir de possible compatibilité au sujet d'autres types de contentieux, et notamment d'atteintes aux personnes. Une polémique publique très révélatrice fut à ce sujet déclenchée, fin 1999, s'agissant de comprendre l'écart considérable entre victimations déclarées et enregistrements policiers au sujet des «injures et menaces». 0 n sait qu'elles sont vécues par les victimes comme des agressions douloureuses, mais que ces faits restent totalement ignorés des statistiques policières, ce qui n'avait rien d'étonnant puisqu'ils ressortent d'actes contraventionnels largement hors champ d'enregistrement de l'index 4001. Or, ce phénomène technique mal expliqué à la presse prit des allures de drame parisien au point qu'il coûta son poste... au directeur de I'IHESI de l'époque, licencié par un ministre furieux d'avoir été mis en position de légèreté par la presse au motif de sous-estimation des chiffres de la délinquance! L'interprétation qui préval ut largement dans les médias en cette occasion était que la police ne donnait pas suite à une très grande partie des faits de délinquance signalés, alors qu'une précaution élémentaire eût été d'expliquer les mécanismes techniques de cette entrave. Est-il besoin d'épiloguer sur le fait que les enquêtes de victimation nationale ne seront pas durablement cotées dans le monde policier français si elles ne devaient servir qu'à révéler leur performance en terme d'élucidation des affaires. Les collectivités territoriales acceptent toutefois aujourd'hui de prendre le relais en finançant plus volontiers des enquêtes locales de victimation (Lagrange et Pottier, 2000) que les analystes mobilisés ont réussi à faire reconnaître comme autant d'intéressantes sources alternatives de connaissance, non pas du «chiffre noir » de la criminal ité réelle, mais de l'utilité de mieux comprendre les stratégies des victimes à l'égard de la police ou autres (Zauberman et Robert, 1995 ; Robert et al., 2001).

D e façon générale, on peut espérer qu'un rapprochement des points de vue sur le bon usage des indicateurs serait possible si chacun y mettait un peu du sien. Certains spécial istes seraient même désormais prédisposés à changer leur prévention à l'égard des usages de la statistique annuelle de la délinquance si l'administration policière faisait preuve de son côté de plus de courage en facilitant la divulgation de ses statistiques local es afin de mieux interpréter les zones de compatibilités des contentieux avec les enquêtes locales de victimation ou, mieux encore, des mesures de l'impact des actions policières dans le cadre de stratégies de rapprochements avec la population (sur la justice, voir Wyvekens et Faget, 2001). 
Q uoi qu'il en soit, la toute-puissante administration policière préfère encore largement sacrifier à deux autres modalités de collectes en accord avec le politique: se fabriquer elle-même ses propres outils de connaissance des violences, ou procéder à des sondages maison ou contrôlés au sujet des réformes mises en place. 0 n peut lire par exemple l'invention de la célèbre «échelle des violences urbaines» par la cellule de la DCRG précitée (Bui-Trong, 2000), non pas comme un instrument prédicteur d'émeutes urbaines futures (à l'épreuve de la réal ité, cette vue a fait long feu), mais comme un instrument ayant légitimé ex post antel'existence de «quartiers sensibles» avec des «populations à risques sui generis» sur lesquelles devait s'intensifier une surveillance policière par définition défaillante. « Populations à risques collectifs» que l'index 4001 ne pouvait objectiver de son côté, entièrement absorbé qu'il demeure par la saisie des données de faits individuels ou de «mis en cause» uniquement compatibles avec les logiques d'emboîtement des catégories judiciaires du Code pénal. Q uant aux sondages «politiques» relatifs à la progression du sentiment d'insécurité rituellement commandités par le ministère (par exemple CRED OC, 2000), ils ont beau avoir montré récemment que la première préoccupation des Français était devenue l'insécurité devant le chômage, la volonté politique du gouvernement socialiste de ne pas dramatiser cette question (pour ne pas parler d'auto-intoxication sur la prétendue satisfaction populaire à l'égard de la police de proximité; voir Institut BVA, 2002) le prit de court deux mois plus tard lors de l'électrochoc constitué par le résultat des élections présidentiel les où l'on vit arriver, contre toute attente, le candidat du Front national au deuxième tour de scrutin.

Comment et pourquoi rapprocher les sources de connaissance quantitatives de la criminalité recueillies de façon hétérogène quand la pression européenne et locale y invite, et que révèle concrètement cet exercice quand on s'efforce de le prendre au sérieux? Cette question fut au cœur du rapprochement qui s'est opéré ces dernières années entre le ministère de l'Intérieur et celui de l'Équipement, pour œuvrer à une politique concertée d'aménagement et d'urbanisme intégrant une meilleure sécurité (Akrich et al., 2001). Un bilan critique des différents outils de mesure disponibles (0 cqueteau et al., 2002) fit ressortir la très grande hétérogénéité des catégories utilisées dans les sources statistiques inventoriées et les surdéterminations implicites de leurs constructeurs en termes de prévention versus répression de faits, d'auteurs ou de comportements. N ous montrions comment le rapprochement des indicateurs restait tributaire des modalités de répartition des compétences en matière de sécu- 
rité, de la propension différentielle des acteurs enrôlés comme coproducteurs de sécurité à se laisser convaincre ou non d'intégrer de nouvelles formes de contrôle social dans leurs pratiques professionnelles (transporteurs, centres commerciaux, établissements scolaires, bailleurs de l'habitat social...), et surtout l'absence d'un réel consensus sur le périmétrage et le ciblage de politiques de prévention et de sécurité localement concertées entre concepteurs des politiques de la Ville et de l'Intérieur (Anderson, 1998) tant que des ambiguïtés majeures subsistaient sur leurs finalités réelles.

\section{I mporter des leçons évaluatives de l'efficacité policière en réduction du crime?}

\section{Heureux monde anglo-saxon...}

Les « indicateurs de performance» quantitatifs d'une police de proximité réformée sont un autre des enjeux les plus intéressants de la dernière décennie. 0 n sait que les indicateurs de l'action préventive ne sont pas faciles à penser et à mettre en œuvre, puisque lorsqu'on arrête- par le hasard du flagrant délit - un auteur, on élucide l'affaire signalée par une ou plusieurs victimes ou l'affaire et l'auteur simultanément. 0 n imagine facilement que la police ne sait pas vraiment ce qu'une action de prévention ou en résolution de problème a évité comme déviances ou délits, à la grande différence des organisations privées qui contrôlent de façon rigoureuse leur «territoire de vie économique» en calculant périodiquement l'étendue de leurs pertes en rapport aux coûts d'investissements consentis pour les enrayer. Policièrement, on reste donc condamné à spéculer sur l'efficacité de la prévention générale sans véritablement réussir à en démontrer l'impact avec des indicateurs ad hoc. Sauf à constater avec le 4001 une baisse tendancielle des signalements de tels types de délits ou crimes, ce phénomène de baisse peut faire l'objet d'interprétations différentes: ou bien plutôt une plus grande désaffection du public à l'égard de la police (puisqu'en bonne logique une hausse de satisfaction devrait plutôt se traduire par une augmentation des faits signalés et rapportés) ou plus vraisemblablement une prise en charge alternative par d'autres acteurs de régulation, telle par exemple la sécurité privée dans les espaces commerciaux à l'égard du vol à l'étalage (0 cqueteau et Pottier, 1998).

N ous n'en sommes pas arrivés en France aux méta-évaluations du style de celle qui fut remise au « $N$ ational Institute of Justice» des États- 
Unis par Law rence Sherman et ses collègues (Sherman, dans Sherman et al., 1997) et retravaillées au Royaume-U ni par P. Jordan (1998), ne seraitce que pour de simples raisons d'investissements financiers sans aucune comparaison possible (Brodeur, 2001). Ces sources nous semblent pourtant des incontournables autorisant à fantasmer sur les orientations à prendre si l'on voulait se donner les moyens de mesurer les performances de l'institution par rapport aux nouvelles doctrines d'emploi de la police en matière de prévention des manifestations de l'insécurité. Pas d'autres moyens alors que de tester dans ces célèbres travaux les hypothèses indécidables faute d'avoir, dans ces pays, été suffisamment documentées, et d'essayer de les acclimater bien modestement à notre horizon hexagonal. On se rappelle que la méta-évaluation de Sherman séparait les hypothèses prouvées des non prouvées au sujet de onze stratégies policières, préventives, répressives ou mixtes, de lutte contre la criminalité. Et cela à partir de tests de corrélation simples entre cause et effet, des mesures de l'avant et de l'après-mise au point de stratégies policières sans ou avec groupe de contrôle, groupes spécifiques ou indifférenciés, ou encore par le biais d'expérimentations au hasard ou contrôlées. 0 r, ni les évaluations américaine et anglaise ne sont parvenues à prouver qu'un accroissement systématique ou à l'aveugle de la densité de la présence policière faisait décroître les taux de criminalité dans l'espace public, parce que pas assez de variables intermédiai res à définir y furent testées. Elles ne parvenaient pas non plus à le montrer, ni au sujet des stratégies d'intensification des contacts informels avec les jeunes, ni au sujet des stratégies d'amélioration de la rapidité d'intervention sur les appels de victimes (sauf pour les cambriolages au Royaume-Uni). Ce qu'elles parvenaient à prouver, en revanche, était qu'un ciblage de l'action auprès de victimes à répétition réduisait leur victimation, de même qu'une présence de patrouilles sur les points ou créneaux horaires chauds, ou encore au sujet d'un travail systématiquement orienté vers des partenariats les plus divers, les échanges d'informations améliorant sensiblement en retour les taux de résolution des faits signalés en terme d'arrestations. L'amélioration de la qualité des contacts entretenus avec la population en général en ressortait comme étant une stratégie insuffisante, même si des indicateurs pouvaient montrer un retour de la confiance populaire à l'égard de la police. Si par ailleurs un ciblage de l'action sur des types de criminels spécifiques semblait avoir un impact sur la diminution de leur récidive aux États-Unis, ce phénomène ne semblait pas vérifié au Royaume-Uni, à l'égard notamment du trafic de drogue dans la rue. Les éval uations anglaises se singu- 
larisent enfin des américaines sur le fait qu'arrêter plus systématiquement les auteurs de crimes signalés ferait chuter la criminalité, évaluations validées en matière de délinquances intrafamiliales mais contestées en matière de délinquance juvénile. Pas d'impact vraiment concluant dans un cas ou dans l'autre au sujet des hypothèses de H. Goldstein, qui prétendait qu'une police orientée vers la résolution des problèmes par identification et réduction de leurs causes les plus immédiates faisait diminuer le crime (contra les évaluations de l'équipe de Braga à Jersey City; Braga et al., 1999).

\section{Qu'en importer?...}

O n en vient donc à tirer trois leçons de cette plongée sur la prévention de la délinquance à partir des usages et actuelles stratégies policières françaises: intuitivement, on peut admettre comme fondée l'idée qu'un retour à la sécurité objective ou de diminution de l'insécurité subjective devrait passer par une stratégie de coconstruction de la prévention des désordres en partenariat dans certains espaces particulièrement insécurisés; qu'il ne pourrait s'obtenir sans des actions ciblées auprès de victimes à répétition et sur des foyers temporaires de troubles; et que le harcèlement auprès de certaines catégories de jeunes dans l'espace public ne fera qu'envenimer les conflits si la seule réponse possible devait se manifester par une répression policière systématique. Vu du côté français, on peut retraduire ces leçons de la façon suivante: si le sentiment d'insécurité est associé à la montée des incivilités chez des jeunes dans les espaces urbains, il est illusoire de s'imaginer que la régulation relèverait prioritairement d'une action policière agressive de type tolérance ou impunité zéro: on ne fera pas l'économie d'enrôler les famille, école et secteur associatif de prévention (Esterle H edibel, 2002); la prévention des victimations à répétition de violences n'aura des chances d'avoir un réel impact que si la police joue un rôle de «médiateur » civique et si son action est correctement relayée par la neutralisation judiciaire des «noyaux durs violents»; quant aux agressions physiques dans l'espace public, enfin, seule la police publique sera fondée à les contenir à condition que la société ne la laisse pas harceler les seules catégories de population prolétarisées, ethnicisées et ségréguées, en laissant croire que seule la répression de leurs rejetons pourrait mettre un terme à la «culture de l'excuse». 
D ans tous les cas de figure, s'agissant du travail policier, il faut compter avec les enseignements de ce qu'un spécialiste du travail policier évoque au sujet de la hiérarchie interne (M onjardet, 1996), à savoir qu'en dépit des réformes actuelles - et cela vaudrait aussi pour la réforme à M ontréal, au Q uébec - les deux indicateurs sur lesquels continuera de veiller jalousement la Direction de la police nationale resteront la tenue de l'index 4001 de la statistique locale de la délinquance et le chiffre des «rappels», c'est-à-dire la tenue rigoureuse de l'état des heures supplémentaires demandées aux agents. 0 r, si I'on essaie d'obtenir simultanément une réduction significative du niveau général de la délinquance, d'obtenir de meilleures relations entre la police et la population et que I'on vise à satisfaire des objectifs internes (rational isation des tâches par redistribution polyvalente des rôles pour limiter les «rappels»), il y a de fortes chances de croire qu'au moins l'un de ces trois objectifs restera sacrifié au détriment des deux autres. Sans vouloir jouer les C assandre, on peut penser que c'est l'objectif de mise en confiance des habitants qui sera le premier rangé au magasin des accessoires car, à la différence de ce qui a pu être évalué à Chicago, personne, ni parmi les partenaires signataires des C.L.S. (Donzelot et al., 2003: 291 et suivantes), ni au sein de la police ni chez les élus, ne croit réellement en France à la mobilisation de «la population» ou «des habitants».

\section{Premières évaluations d'efficience et d'efficacité de la réforme en cours}

Le préalable indispensable à la « réussite» de la réforme de la police de proximité a paru résider dans une recherche action que nous lançâmes avec D. M onjardet, visant à expliquer les voies d'une transmission de la doctrine officielle dans les écoles de police par la valorisation des savoirfaire policiers préexistants à partir d'une suggestion de M astrofski (1998). L'enquête de S. Tiévant (2001), aidée par la cueillette des témoignages sur le «mystère» de policiers, dans l'environnement de différentes circonscriptions de police où ils travaillaient de longue date, réputés réussir mieux que certains de leurs collègues non pas à résoudre les tensions sociales locales mais au moins à les diminuer, permit à cette auteure de monter le phénomène en généralité stratégique. Elle montra comment sept des grands principes guidant le «nouveau » travail policier organisaient son action en développant un schéma en trois composantes. Par un effet de consensus sur l'identification et la pertinence des 
ressources (savoirs théoriques, compétences, qualifications, techniques et savoir-faire) à déployer sur le terrain, personne ne trouva à redire à ce référentiel, ni à son principe d'efficacité, ni aux principes des modalités pratiques de sa mise en œuvre, autrement dit les trois ingrédients indispensables à la réussite de toute réforme, puisque les germes de la réussite étaient virtuellement contenus dans plusieurs exemples concrets aisément puisables dans ce travail. S'agissant du référentiel, l'auteure montre par exemple qu'il est toujours plus difficile à un jeune de lancer des pierres sur un policier qui le connaît et dont il est connu que sur un policier anonyme. S'agissant du principe d'efficacité, qu'un policier qui connaît un maximum de personnes par leur nom ou surnom et se montre capable de témoigner en toutes occasions qu'il les connaît et peut les nommer a déjà beaucoup gagné en crédibilité. S'agissant de la mise en œuvre enfin, elle montre qu'une fidélisation réelle au quartier ou à l'îlot passe par une circulation pédestre incessante destinée à se faire connaître en renonçant à son anonymat, en distribuant des cartes de visite pour ne jamais laisser des demandes insatisfaites même si elles ne peuvent toutes être résolues par le seul policier de proximité (dans le même sens, aux Pays-Bas, van der Vijver, 2000). Ces prérequis sont actuellement en cours d'actualisation par un nouveau diagnostic d'évaluation de la même auteure auprès de "partenaires» (associatifs, transporteurs, commerçants...) censés améliorer la situation dans le même environnement pour ne pas laisser penser que l'impact réel de la réforme ne dût passer que par une stratégie unilatérale de la police. II s'agit en effet de détecter à quelles conditions systémiques de tels acteurs se mobilisent ou non, à proportion de la façon dont ils voient s'engager elle-même la police de proximité sur leur terrain. S. Tiévant (2002a; 2002b) vient de mettre à jour à ce sujet une intéressante typologie de trois types d'engagements dans le partenariat avec la police que tous les futurs évaluateurs, internes ou externes, de l'efficacité de ce type de réforme à long terme gagnerai ent à méditer.

M a propre implication sur l'évaluation de l'efficience a trouvé à s'exercer avec Jérôme Ferret (2001), chercheur avec lequel nous fûmes enrôlés comme enquêteurs avec les agents d'inspection de la police nationale. Elle eut pour but d'évaluer la bonne marche de la réforme dans trois des 65 premières circonscriptions. En nous concentrant sur trois villes, nous avons documenté un certain nombre de prérequis. Le protocole que nous nous étions fixé en cette occasion est un classique de la sociologie des organisations en mouvement (Gatto et Thoenig, 1993), centré sur la détection des résistances à l'innovation au moyen d'une compréhension 
générale du contexte local et de son «système d'action concret» au sein de ces trois villes. II s'agissait de pénétrer au cœur de la boîte noire, en dressant un inventaire complet des «intérêts» défendus localement par le préfet ou son chef de cabinet, du procureur ou de son substitut, des services du maire et de son chargé de mission sécurité, et de ceux du commissaire de police. À l'égard de ce dernier, nous voulions observer la façon dont il s'émancipait ou se conformait aux recommandations du diagnostic du contrat local de sécurité au vu des contraintes organisationnelles pesant sur son service et des marges de manœuvre disponibles pour mobiliser ses troupes conformément aux injonctions centrales (voir également, sur la mobilisation des adjoints de sécurité, Gorgeon, 1999).

Vu la rapidité de la démarche, nous n'avons évidemment pu faire ressortir que des impressions dont nous avons tenté rétrospectivement de tester la solidité auprès d'autres analystes au cours d'un séminaire de chercheurs portant sur d'autres évaluations de villes, voire des mêmes (Gorgeon, 2001), et en discutant de nos conclusions avec les acteurs engagés et analystes locaux. J. Ferret (2002 : 107-116) ayant déjà rendu amplement compte des résultats, je me contenterai ici d'en rappeler les leçons principales en problématisant l'enjeu de la façon suivante: "à quelles conditions une dynamique globale peut-elle s'enclencher pour que des résultats significativement différents apparaissent concrètement sur le terrain, dans les pratiques policières et les représentations de ces différents acteurs?». Cette formulation hypothético-déductive me semble plus exacte que la vue rétrospective de l'impact sur la réduction locale du crime, impact que la nouveauté de la réforme ne permet pas de documenter pour l'instant. II en est ressort au moins quatre enseignements:

- Premier enseignement: la variable du «climat du système politique local » apparaît comme la plus importante de toutes les variables pour produire ou non un effet significatif de la réforme de la police de proximité. Dans l'une des villes étudiées, la densité du réseau associatif de prévention bien maîtrisé par le maire et le dynamisme de son service social, la faiblesse des effectifs de sa police municipale, et le calcul consistant pour des opposants politiques à jouer le jeu de voir des policiers se redéployer différemment sur une portion problématique du territoire laissée de longue date aux routines d'une police d'État peu performante, leur interdisaient de contester une expérience qu'ils espéraient voir pérennisée après les élections locales dont ils pouvaient tirer bénéfice. $D$ 'autant que, de son côté, le commissaire de police se trouva libre de ses mouvements par un préfet et un procu- 
reur pour qui l'enjeu était faible, s'agissant de la pression criminelle. Les échanges d'informations entre nouvelle police de proximité et acteurs sociaux ne pouvaient que susciter un cercle vertueux de bonne reprise en main du quartier insécurisé.

- Deuxième enseignement: dans une localité où la pression criminelle était plus forte, la configuration se présenta de façon sensiblement différente. Le clivage des intérêts politiques défendus entre l'élu et le préfet était beaucoup plus pesant. Au point qu'on put observer deux politiques parallèles concurrentes et augurer de leur contre-productivité. Le chef du cabinet du préfet tentait en effet d'imposer autoritairement la réforme dans l'espoir de faire gagner l'opposant politique du maire en place en faisant vivre des cellules de veille remplies par les agents locaux de médiation sociale (ALM S) dont, de son côté, le maire ne savait que faire, la préfecture faisant remonter de l'information sur la gestion municipale sous couvert de ces missions de «veille sociale». Les cadres intermédiaires de l'équipe de police, renouvelée à grand renfort de mobilisation de nouveaux adjoints de sécurité, jouaient de leur côté leur propre partition en partenariat avec les services techniques de la ville, en raison de la longue complicité qui les unissait avec l'équipe municipale sur le terrain du quartier très problématique de cette ville. Et cela au détriment du nouveau commissaire de police qui, faute de légitimité suffisante, se montra désinvesti et incapable de répondre aux injonctions du chef de cabinet du préfet. La position des policiers se trouva par ailleurs confortée par celle du substitut du procureur qui sut habilement anticiper les effets induits de la réforme.

- Ce qui conduit au troisième enseignement. 0 n sait que le parquet est un acteur en général intéressé par le seul travail de la police judiciaire: des affaires bien ficelées par la police doivent lui permettre de poursuivre et faire traduire en justice des délinquants chevronnés. Les procureurs passent en général pour plutôt désinvestis dans les politiques de justice de prévention et de proximité. Dans le cas de la ville précédente, on observa néanmoins une sorte d'exception à cette règle. En effet, «la variable judiciaire » y fut une autre condition du «succès». Le déploiement de la police de proximité provoqua un effet non attendu d'accroissement du contentieux $d^{\prime}$ " outrages et rébellion à l'égard de la force publique», tendance confirmée ailleurs. Ce qui était sociologiquement assez logique, vu qu'occupant plus systématiquement le terrain la nouvelle police gêna les populations réputées inciviles, et pour s'imposer aux perturbateurs qui avaient de facto 
pris I'habitude d'occuper abusivement l'espace public à leur seul profit, dut en passer par la menace de faire sanctionner les marques de défi à son autorité. $0 \mathrm{r}$, il s'agissait de montrer que la justice pouvait se solidariser avec les nouveaux intérêts de cette police engagée dans la reconquête d'un «territoire» estimé perdu. Le procureur comprit en l'espèce la teneur de cet enjeu symbolique en acceptant de changer l'ordre de ses priorités répressives locales. À cet égard, nous avons pu observer une profonde division des représentations et des pratiques selon les villes, les commissaires de police n'adoptant pas, ni ne justifiant, les mêmes stratégies: les uns plaident qu'un usage trop fréquent de l'arme juridique de l'outrage et de la rébellion est une marque de faiblesse policière dans la mesure où elle laisserait à la justice le soin d'arbitrer entre «jeunes» et «nouveaux policiers», ce qui diminuerait dangereusement les capacités d'initiative et le savoir-faire des agents expérimentés. Pour d'autres, ce serait une nécessité: toutes les armes seraient bonnes à prendre dans la dramaturgie de la « reconquête du territoire» contre ses occupants illégitimes. II me semble en réalité que la variable discriminant ces deux attitudes soit explicable par deux facteurs: une estimation policière de la relativité de la «pression criminelle» et de ses manifestations en terme d'insécurité subjective sur tel quartier; et/ ou un calcul sur l'utilisation des nouveaux «adjoints de sécurité» encadrés, très peu assurés de leur légitimité professionnelle, et qu'il faut convaincre et rassurer en vue d'une stratégie d'incorporation future dans la «maison police».

- Dernier enseignement plus prospectif : il suffit d'identifier l'ensemble des facteurs de blocage énumérés ci-dessus, et d'étudier la volonté et la capacité collective à les résoudre pour prophétiser le succès ou l'échec de l'impact d'une «police de proximité» sur le crime ou l'insécurité. L'échec sera certain si on cherche à expérimenter à tout prix cette nouvelle police dans les quartiers où les probabilités de «violences urbaines» sont maximales. N ous n'avons d'ailleurs pas été longs à nous rendre compte que, dans une ville connaissant des quartiers très différents à ce sujet, le déploiement d'une police de proximité était plus aisé là où il ne se passait quasiment rien, tandis qu'à l'autre extrémité de la ville le commissaire, réaliste, à tort ou à raison, ne tenta pas vraiment de la mettre en œuvre. D e son point de vue, cette attitude eut été suicidaire car elle eut été vécue comme une provocation et une inutile mise en danger de ses nouveaux agents, ce qui conforte d'ailleurs l'observation précédente. 


\section{Pour conclure...}

Est-ce à dire que cette réforme ambitieuse serait d'ores et déjà vouée à l'échec pour ne pas dire condamnée dans I'œuf? D'aucuns le pensent, et notamment tous ceux qui ont intérêt à ne pas croire aux conditions de sa relative réussite, parce qu'il n'existerait pas de réels indicateurs politiquement lisibles de cette "réussite». Les sociologues se sont même trompés sur les sanctions des électeurs aux élections municipales de mars 2001 à partir de villes où certains avaient pu diagnostiquer des «cercles vertueux » ou « vicieux » en matière de coproduction de sécurité collective par le biais de la mise en œuvre de cette «nouvelle police de proximité», alors que tout portait à croire que la « sécurité des personnes et des biens» y était devenue le premier enjeu local.

Un indice aurait dû mettre la puce à l'oreille. Une enquête conduite par un chercheur (M ouhanna, 2000) à six mois d'intervalle sur la population de deux villes pilotes, au moment de l'affichage local de la réforme et une fois effectuée la mise en place du redéploiement policier, montra que la virulence de la population dans ses critiques ou attentes à l'égard de la police avait pu s'estomper dans l'une d'elles. M ais que pour la population d'un quartier difficile de l'autre, et notamment pour les jeunes de ce quartier (17-25 ans), les opinions apparurent beaucoup plus tranchées et mieux argumentées: la «nouvelle police» était ressentie comme beaucoup plus proche des intérêts des commerçants et systématiquement plus intolérante à leur égard. Surtout, ce chercheur diagnostiqua un net effet de frustration d'une partie de la population, le sondage sur les attentes populaires ayant montré la seconde fois une plus grande proportion de déçus par rapport à la première consultation, cette déception s'expliquant par l'effet d'anticipation selon lequel les missions de contact police/ populations s'élargissant, les actions semblaient pour ceux qui y avaient mis quelque espoir, à tort ou à raison, peu suivies d'effets réels.

Ce que le sociologue pouvait analyser comme un effet pervers, ou comme la preuve d'un «succès» au sens où quelque chose s'était passé dans la population, fut en l'occurrence violemment récusé par le cabinet du ministre et le commissaire concernés. Ces acteurs admirent difficilement de voir ce type d'évaluation «non contrôlée» se répandre à un moment où il fallait politiquement faire état d'une réussite globale de la réforme. Anecdote significative de ce déni de savoir : le cabinet du ministre s'empara du questionnaire de ce sociologue indépendant et confia à partir de cette grille de cueillette à des « sondeurs» encadrés, de 
nouvelles consultations en une seule fois dans d'autres agglomérations, et notamment à Paris, sondages dont les résultats ne furent évidemment jamais rendus publics.

Difficile dans ces conditions, lorsqu'on est au milieu du gué et au cœur de la boîte noire, de rester idéaliste. Le «réalisme» doit rendre moins naïf. Les configurations locales sont complexes, les intérêts en jeu très pesants. II n'y a pourtant aucune raison de réduire les questions de l'évaluation du travail policier à la seule dimension «réduction du crime» dans la mesure où l'objectif affiché de la réforme, comme celle des C.L.S., était d'agir autant sur la réduction du sentiment objectif que subjectif de l'insécurité. La nécessité d'éval uer l'impact de ces réformes demeure, mais il me semble impératif de ne jamais oublier de décrire et de méditer les enjeux et effets sociopolitiques rétroactifs, nationaux et locaux, du comportement des acteurs collectifs metteurs en oeuvre, première exigence de lucidité analytique.

\section{Références}

A krich, M ., M éadel, C., Frenais, J., \& 0 cqueteau, F. (2001). D ésordres en territoires urbains, pour une confrontation des positions. Les cahiers de la sécurité intérieure, 43, 81-100.

Anderson, A. (1998). Politiques de la ville, de la zone et du territoire. Paris: Syros.

Becker, H.S. (2002). Les ficelles du métier, comment conduire sa recherche en sciences sociales. Paris: La D écouverte.

Bègue, L. (2000). A ttachements sociaux, croyances conventionnelles et délinquance. Paris: IHESI, études et recherches.

Brodeur, J.-P. (2001). La recherche institutionnelle en matière de justice pénale aux Etats-Unis. Les cahiers de la sécurité intérieure, 46, 167-196.

Braga, A.A., Weisburd, D.L., Waring, E.J., M azerolle, L.G., Spelman, W., \& Gajewsky, F. (1999). Problem-solving in violent crime places: a randomized controlled experiment. Criminology, 37 (3), 541-580.

Bui Trong, L. (2000). Violences urbaines, des vérités qui dérangent. Paris: B ayard.

Carraz, R., \& H yest, J.-J. (1998). R apport au premier ministre sur une meill leure répartition des effectifs dela police et de la gendarmerie pour une meilleure sécurité publique. Paris, avril.

Chalumeau, E., \& Glorieux, P. (1999). L'expertise en matière de sécurité. Revue française d'administration publique, 91, 399-412.

Craw ford, A. (1997). The local governance of crime: appeals to community and partnerships. 0 xford: Clarendon.

CRED OC (2000). Les opinions sur la sécurité et les forces de l'ordre au début 2000. Paris: IHESI, études et recherches.

Donzelot, J., M evel, C., \& Wyvekens, A. (2003). Faire société, la politique de la veille auX États-U nis et en France. Paris: Le Seuil. 
Esterle Hedibel, M . (2002). Jeunes des cités, police et désordres urbains. In L. M ucchielli \& P. Robert (eds), C rime et sécurité, l'état des savoirs (376-385). Paris: La D écouverte.

Ferret, J. (2001). Police de proximité en France, une expérience de recherche institutionnelle à l'IHESI entre 1998 et 2001. Les cahiers dela sécurité intérieure, 46, 97-118.

Ferret, J., \& M affre, P. (2000). L'usage de la notion de police de proximité en Espagne: indice d'une mutation inachevée. Les cahiers de la sécurité intérieure, $39,77-102$

Ferret, J., \& 0 cqueteau F. (1998). Évaluer la police de prox imité? Problèmes, concepts, méthodes. Paris: La D ocumentation française.

Gatto, D., \& Thoenig, J.-C. (1993). La sécurité publique à l'épreuve du terrain. Paris: IHESI-L'H armattan.

Grémy, J.-P. (2000). M esurer la délinquance à partir du témoignage des victimes, I'enquête de victimation IN SEE-IHESI 1999. Paris: IHESI, études et recherches.

Gorgeon, C. (1999). Les A d S dans la police nationale. Entrel'institution policière et la population : un effet pervers. Paris: IHESI, études et recherches.

Gorgeon, C. (2001). Existet-il des systèmes locaux de sécurité? Paris: IHESI, études et recherches.

Institut B.V.A. (2002). Police de proximité: principaux enseignements de l'étude. BVA A ctualités, 22 février, www.bva.fr.

Jobard, F. (2002). Bavures policières? La force publique et ses usages. Paris: La D écouverte.

Jordan, P. (1998). «Effective policing strategies for reducing crime». In P. Goldblatt $\&$ C. Lewis (eds), Reducing offending : an assessment of research evidence on ways of dealing with offending behaviour (63-82). Londres: Home O ffice, Research and Statistic Directorate.

Lagrange, H., \& Pottier, M.-L. (2000). U ne enquête de préfiguration sur les risques urbains à A miens. Paris: CESD IP, études et données pénales.

M astrofski, S. D. (1998). Community policing and police organization structure. In J.-P. B rodeur (ed.), H ow to recognize good policing, problems and issues (161192). Londres: Sage.

M inistère de l'Emploi et de la Solidarité, ministère délégué à la Ville, ministère de la Justice, ministère de l'Éducation nationale, ministère de l'Intérieur, ministère de la Défense, Forum français pour la sécurité urbaine (1998). $G$ uide pratique pour les contrats locaux de sécurité. Paris: La D ocumentation française.

M inistère de I'Intérieur (2000). G uide pratique de la police de prox imité. Paris: La Documentation française.

M onjardet, D. (1996). Ce que fait la police, sociologie de la force publique. Paris: La Découverte.

M ouhanna, C. (2000). Q uel service pour quel public? U ne tentative d'éval uation chiffrée de l'image de la police dans la population face à la territorialisation. Paris: IHESI, études et recherches.

0 cqueteau, F. (2002). Évaluation des recherches financées par I'I H ESI 1998-2001. Paris: IHESI, études et recherches. 
O cqueteau, F., Frenais, J., \& Varly, P. (2002). 0 rdonner le désordre, une contribution au débat sur les indicateurs du crime. Paris: La D ocumentation française.

0 cqueteau, F., \& Pottier, M.-L. (1998). Comment règle-t-on le vol à l'étalage dans les espaces commerciaux français? Criminologie, Xxx। (2), 69-85.

Pierre, C. (2001). La sécurité intérieure, sait-on ce que coûte la sécurité? A dministration, 191 (3), 55-61.

Robert, P. (1977). Les statistiques criminelles et la recherche: réflexions conceptuelles. D éviance et société, I (1), 3-27.

Robert, P., Zauberman, R ., Pottier, M.-L., \& Lagrange, H. (2001). M esurer le crime: entre statistiques de police et enquêtes de victimation 1985-1995. Revue française de sociologie, 42, 133-175.

Roché, S. (2001). La délinquance des jeunes, les 13-19 ans racontent leurs délits. Paris: Seuil.

Sherman, L.W. (1997). Policing for crime prevention. In L.W. Sherman, P. Gottfredson, D. M cKenzie, J. Eck, P. Reuter \& S. Bushway (eds), Preventing crime: what works, what doesn't, what's promising? ( $8^{e}$ chapitre). Washington, DC : US D epartment of Justice, 0 ffice of Justice programs.

Tiévant, S. (2001). Les savoir-faire en police de prox imité. Paris: IHESI, études et recherches.

Tiévant, S. (2002a). Caractérisation des savoir-faire spécifiques en police de prox imité et ex amen des moyens de leur acquisition. Paris: IHESI, études et recherches.

Tiévant, S. (2002b). Partenariat et police de proximité. Dilution ou consolidation des spécificités professionnelles? Les cahiers de la sécurité intérieure, 48, 149-170.

Van der Vijver, K. (2000). La police de proximité aux Pays-Bas: le cas de la ville de $H$ aarlem. Les cahiers de la sécurité intérieure, 39, 29-56.

Zauberman, R., \& Robert, P. (1995). D u côté des victimes, un autre regard sur la délinquance. Paris: L'H armattan.

Wyvekens, A., \& Faget, J. (2001). La justice de prox imité en Europe, pratiques et enjeux. Ramonville Ste-Agne: Erès. 